\title{
Peptidoglycan recognition proteins in Drosophila immunity
}

\author{
Shoichiro Kurata* \\ *To whom correspondence should be addressed. \\ Tel.: +81-22-795-5916 \\ Fax: +81-22-795-6802 \\ E-mail: kurata@mail.pharm.tohoku.ac.jp
}

Graduate School of Pharmaceutical Sciences, Tohoku University, Sendai 980-8578, Japan

Abbreviations: LPS, lipopolysaccharides; PGN, peptidoglycans; GlcNAc, $N$-acetylglucosamine; MurNAc, $N$-acetylmuramic acid; DAP, diaminopimelic acid; TLR, Toll-like receptor; NOD, nucleotide-binding oligomerization domain-containing protein; PGRP, peptidoglycan recognition protein; proPO, prophenoloxidase; PPAE, proPO activating enzyme; GNBP, Gram-negative binding protein; SPE, Spätzle processing enzyme; TCT, tracheal cytotoxin; RIP, receptorinteracting protein; RHIM, RIP homotypic interaction motif 


\begin{abstract}
Innate immunity is the front line of self-defense against infectious non-self in vertebrates and invertebrates. The innate immune system is mediated by germ-line encoding pattern recognition molecules (pathogen sensors) that recognize conserved molecular patterns present in the pathogens but absent in the host. Peptidoglycans (PGN) are essential cell wall components of almost all bacteria, except mycoplasma lacking a cell wall, which provides the host immune system an advantage for detecting invading bacteria. Several families of pattern recognition molecules that detect PGN and PGN-derived compounds have been indentified, and the role of PGRP family members in host defense is relatively well-chacterized in Drosophila. This review focuses on the role of PGRP family members in the recognition of invading bacteria and the activation and modulation of immune responses in Drosophila.

Keywords: Pattern recognition receptor, Peptidoglycan recognition protein, Antimicrobial defense, Prophenoloxidase cascade, Innate immunity, Drosophila, Antimicrobial peptides, Self non-self recognition
\end{abstract}




\subsection{Peptidoglycans as a target for immune response}

Innate immunity is the front line of self-defense against infectious non-self in vertebrates and invertebrates (Akira et al., 2006; Hoffmann and Reichhart, 2002). The innate immune system is mediated by germ-line encoding pattern recognition molecules (pathogen sensors) that recognize conserved molecular patterns present in pathogens but absent in the host, such as lipopolysaccharides (LPS) of Gram-negative bacteria, lipoteichoic acids of Grampositive bacteria, peptidoglycans (PGN) of Gram-positive and Gram-negative bacteria, $\beta$-1,3glucans of fungi, and DNA and RNA of bacteria and viruses (Akira et al., 2006; Medzhitov and Janeway, 2002). After ligand recognition, pattern recognition molecules activate or modulate various immune reactions, including acquired immunity in vertebrates (Akira et al., 2006; Lemaitre and Hoffmann, 2007). Although LPS is a potent immune stimulator in vertebrates and invertebrates, it has no immune stimulating activity in systemic humoral responses in the fruit fly, Drosophila melanogaster, a good model organism for studying the basic principles of innate immunity using genetic and molecular biology techniques (Akira et al., 2006; Lemaitre and Hoffmann, 2007). In contrast to LPS, PGN of Gram-positive and Gram-negative bacteria stimulate multiple immune reactions in Drosophila.

PGN are essential cell wall components of almost all bacteria, except mycoplasma lacking a cell wall. PGN provides the host immune system an advantage for detecting invading bacteria, as the enzymes regulating PGN biosynthesis are excellent targets for many antibiotics. PGN are polymers of $\beta$-1,4-linked $N$-acetylglucosamine (GlcNAc) and $N$-acetylmuramic acid (MurNAc) cross-linked by short stem peptides. The glycan chain is relatively conserved in all bacteria, whereas the amino acid composition of the cross-linking stem peptides and the linkage between stem peptides are diversified depending on the bacterial species (Schleifer and Kandler, 1972). Many Gram-negative bacteria and some Gram-positive bacteria such as the Bacillus species have directly cross-linked PGN containing diaminopimelic acid (DAP) at position three of the stem peptides (DAP-type PGN). Many Gram-positive bacteria, on the other hand, have PGN containing lysine instead of DAP ,with cross-linking peptides between stem peptides (Lystype PGN; Fig. 1). The muramyl dipeptide MurNAc-L-Ala-D-isoGln was first identified as a minimal PGN fragment based on its mammalian immune-stimulating activity as an adjuvant effect from the mycobacterial cell wall, and PGN itself and PGN-derived molecules are potent immune stimulators. Several families of pattern recognition molecules have been indentified to 
detect PGN and PGN-derived compounds, such as Toll-like receptors (TLRs) and nucleotidebinding oligomerization domain-containing proteins (NODs) in vertebrates, and PGN-binding proteins (PGRPs) in vertebrates and invertebrates (Akira et al., 2006; Ferrandon et al., 2007). The role of the PGRP family in host defense is relatively well chacterized in Drosophila (Royet et al., 2011). This review focuses on the role of PGRP family members in the recognition of invading pathogens and the activation and modulation of immune responses in Drosophila.

\subsection{Structure of PGRPs}

PGRP was first purified from the silkworm (Bombyx mori) hemolymph based on its high affinity to bacterial PGN (Yoshida et al., 1996). PGRP mediates PGN-dependent activation of the prophenoloxidase (proPO) cascade, leading to localized wound healing and melanization (Yoshida et al., 1996). Prophenoloxidase is activated to phenoloxidase by the serine protease cascade, including proPO-activating enzyme in the hemolymph. The cytotoxic reactive oxygen species and melanin are produced during the phenoloxidase-mediated conversion of dopamine into melanin, which are thought to be toxic to microorganisms (Soderhall and Cerenius, 1998). The subsequent cloning of PGRP genes demonstrated their conservation from insects to mammals, but PGRP genes are not present in lower metazoan such as Caenorhabditis elegans or plants (Kang et al., 1998; Royet et al., 2011). For example, Drosophila has 13 PGRP genes encoding 19 proteins, mosquito (Anopheles gambiae) has 7 PGRP genes encoding 9 proteins, and humans and mouse have 4 PGRP genes (Christophides et al., 2002; Kang et al., 1998; Werner et al., 2000; Werner et al., 2003). Almost 100 PGRP family members have been identified, and all have at least one conserved PGRP domain similar to $N$-acetylmuramyl-alanine amidases, such as bacterial amidases and bacteriophage lysozymes, suggesting an ancestral origin of this protein family (Kang et al., 1998). In fact, some PGRPs (catalytic PGRPs) are capable of hydrolyzing PGN by cleaving the amide bond connecting MurNAc to Ala of the stem peptide (Mellroth et al., 2003; Royet et al., 2011). Other PGRPs (sensor PGRPs) bind to PGN, but do not hydrolyze PGN because of the lack of a cysteine residue critical for amidase activity (Guan and Mariuzza, 2007; Kang et al., 1998; Royet et al., 2011).

Insect PGRPs are also categorized into two groups based on the transcript size (Werner et al., 2000). Short PGRPs (PGRP-S) have signal peptides and can be extracellular proteins; long PGRPs (PGRP-L) can be intracellular, extracellular, and transmembrane proteins (Fig. 2). The 
structural similarity between the PGRP domain and $N$-acetylmuramyl-alanine amidases was confirmed by crystal structure analysis, which revealed three peripheral $\alpha$-helices and a central $\beta$-sheet with five $\beta$-strands (Guan and Mariuzza, 2007; Kim et al., 2003). The PGN-binding grooves of PGRPs basically bind to fragments of PGN, muramyl pentapeptide or muramyl tetrapeptide, inducing a structural change in the PGRP domain or an interaction with other PGRP molecules, which seems to be important for signaling activation, as described below. In Drosophila, PGRP genes are expressed in immune-reactive tissues, such as hemocytes (blood cells), the fat body (functional equivalent of mammalian liver), the gut, and the epidermis (Werner et al., 2000). The expression of most PGRP-S is upregulated in response to bacterial infection, whereas many PGRP-L are constitutive proteins (Werner et al., 2000).

\subsection{Sensor function of Drosophila PGRPs in immune responses}

An important immune response in insect is the induction of antimicrobial peptides in immune tissues, such as the fat body and epithelial tissues (Ferrandon et al., 2007; Hoffmann and Reichhart, 2002; Lemaitre and Hoffmann, 2007; Steiner et al., 2009). In Drosophila, seven classes of antimicrobial peptides with different target specificities have been identified. For example, Drosomycin is active against fungi, Diptericin is active against Gram-negative bacteria, and Defensin is active against Gram-positive bacteria. These antimicrobial peptides are secreted from the fat body into the hemolymph as a systemic immune response induced by the activation of two distinct NF- $\mathrm{BB}$ signaling pathways, the imd pathway and the Toll pathway. These two $\mathrm{NF}-\kappa \mathrm{B}$ signaling pathways are similar to the mammalian tumor necrosis factor- $\alpha$ receptor signaling pathway and TLR/interleukin-1 receptor signaling pathway, respectively (Hoffmann and Reichhart, 2002; Hultmark, 2003). The Toll pathway is activated predominantly in response to fungal and some Gram-positive bacterial infections and induces antimicrobial peptides such as Drosomycin. The imd pathway is activated predominantly in response to Gram-negative and other Gram-positive bacterial infections, and induces antimicrobial peptides such as Diptericin, indicating that Drosophila distinguishes between different pathogens and activates appropriate immune responses (Ferrandon et al., 2007; Hoffmann and Reichhart, 2002; Lemaitre and Hoffmann, 2007; Lemaitre et al., 1997). Upstream of both the Toll pathway and the imd pathway, PGRP family members act as pattern recognition molecules to distinguish invading bacteria (Fig. 3). 
PGRP-SA was first identified as a sensor PGRP in Drosophila, required for activation of the Toll pathway in the fat body in response to Gram-positive bacterial infections (Michel et al., 2001). PGRP-SA is a short PGRP circulating in the hemolymph and cooperates with PGRP-SD, another short PGRP, and Gram-negative binding protein (GNBP) 1, another pattern recognition molecule family member, to activate the Toll pathway, as described below (Bischoff et al., 2004; Gobert et al., 2003). For activation of the Toll pathway in the fat body, the serine protease cascade is triggered after PGRP-SA binds to the PGN of Gram-positive bacteria (Lys-type PGN) to convert the inactive ligand for the Toll receptor, proSpätzle, to the active ligand, Spätzle (Spz), through proteolysis by the Spz-processing enzyme (Ferrandon et al., 2007; Jang et al., 2006; Lemaitre and Hoffmann, 2007; Ligoxygakis et al., 2002; Weber et al., 2003). GNBP was also first identified from Bombyx mori hemolymph based on its high affinity to Gram-negative bacteria, but in Drosophila, GNBPs are not involved in the recognition of Gram-negative bacteria (Lee et al., 1996). Another GNBP family member, GNBP3, binds to $\beta$-1,3-glucans of fungi and is required for activation of the Toll pathway induced by fungal cell walls (Gottar et al., 2006). A protease of entomopathogenic fungi, a virulence factor, also activates the Toll pathway by converting Persephone into an active protease that triggers the serine protease cascade to produce active Spz, suggesting a dual detection system for fungal infection by pattern recognition molecules and virulence factors (Gottar et al., 2006).

PGRP-SA and -SD are not involved in the recognition of Gram-negative bacteria and activation of the imd pathway, whereas other PGRP family members, PGRP-LC and -LE, recognize the PGN of Gram-negative bacteria (DAP-type PGN) and activate the imd pathway in the fat body. PGRP-LC is a transmembrane receptor that binds PGN through the extracellular PGRP domain and interacts with Imd, an adaptor protein of the imd pathway similar to mammalian receptor-interacting protein 1 (RIP1), through an intracellular domain, which is required for activation of the imd pathway in response to Gram-negative bacterial infections (Choe et al., 2002; Gottar et al., 2002; Ramet et al., 2002). PGRP-LE is a long PGRP lacking a signal peptide that is detected in both the hemolymph and cytoplasm of immune-related cells, such as the fat body, gut and hemocytes, although the sorting system of PGRP-LE is unclear (Takehana et al., 2002; Takehana et al., 2004; Yano et al., 2008). PGRP-LE binds to DAP-type PGN and activates the imd pathway in two distinct ways; extracellular PGRP-LE activates the imd pathway at least in the fat body through the PGRP-LC transmembrane receptor, possibly by 
forming the PGRP-LC and -LE complex, whereas intracellular PGRP-LE activates the imd pathway through interactions with Imd by the N-terminal region in PGRP-LC-independent manner, at least in cell culture, as described below (Kaneko et al., 2006; Takehana et al., 2002; Takehana et al., 2004; Yano et al., 2008). The distinct function of PGRP-LE from PGRP-LC is required for the induction of immune responses to infectious bacteria and tolerance to commensal bacteria in the gut (Bosco-Drayon et al., 2012; Neyen et al., 2012). Intracellular bacterial recognition by PGRP-LE acts in concert with the JAK-STAT pathway, another signaling pathway, to induce the antibacterial peptide Listericin in cell culture (Goto et al., 2010). PGRP-LE is the sole intracellular pattern recognition molecule so far identified in Drosophila.

Intracellular bacteria that cause serious infectious diseases, such as tuberculosis, shigellosis, and listeriosis in human, invade cells and are thus able to escape humoral immune responses, such as killing by antimicrobial peptides. In fact, intracellular bacterial growth is not affected by the imd pathway-dependent induction of antimicrobial peptides in Drosophila (Yano et al., 2008). In hemocytes, PGRP-LE recognizes Listeria monocytogenes, bacteria with DAPtype PGN, in the cytoplasm and induces autophagy, a bulk self-degradation mechanism for the turnover of proteins and organelles that is conserved from yeast to humans, to eliminate intracellular bacteria in an imd pathway-independent manner (Fig. 3) (Yano et al., 2008). In mammalian cells, intracellular pattern recognition molecules detecting PGN fragments, NOD1 and NOD2, have a crucial role in the induction of autophagy againt L. monocytogenes and Shigella flexneri infections (Travassos et al., 2010). NOD-dependent activation of autophagy is independent from RIP2, an adaptor protein that activates NF- $\kappa \mathrm{B}$ to trigger proinflammatory signals, and NEMO, an NF- $\mathrm{B}$ essential modulator, suggesting a similarity in the recognition of PGN and the activation of autophagy between insects and mammals.

PGRP was identified as a PGN-binding protein that mediates PGN-dependent activation of the proPO cascade, as mentioned above. Biochemical analysis of the activation mechanisms has progressed in other insects (Kim et al., 2008). In Drosophila, overexpression of PGRP-LE and constitutively activated PGRP-LC activate the proPO cascade (Schmidt et al., 2008; Takehana et al., 2002). PGRP-LE activates the proPO cascade upstream of the proPO-activating enzyme, which is regulated by a serine protease inhibitor, Serpin 27A (Fig. 3) (Takehana et al., 2004). Therefore, PGRP-LE is a multifunctional pattern recognition molecule that induces activation of the imd pathway and the proPO cascade extracellularly, and the imd pathway and 
autophagy intracellularly, which possibly reflects the characteristic of innate immunity that various immune responses are induced by a limited number of germ-line encoded pattern recognition molecules.

How do sensor PGRP members distinguish between invading bacteria to activate the appropriate downstream signaling? As described above, PGN are classified into two major categories, Lys-type and DAP-type PGN, depending on the bacterial species. Drosophila sensor PGRPs distinguish between bacteria based on the structural differences of PGN of the bacterial cell walls. PGRP-LE, a sensor PGRP upstream of the imd pathway, was first demonstrated to bind to the DAP-type PGN but not to Lys-type PGN in vitro (Takehana et al., 2002). In fact, the imd pathway is activated by DAP-type PGN-containing bacteria and DAP-type PGN, but not by bacteria with Lys-type PGN in vivo (Kaneko et al., 2004; Leulier et al., 2003), whereas the Toll pathway is mainly activated by Lys-type PGN and weakly activated by DAP-type PGN (Leulier et al., 2003). The PGRP-LC gene is required for DAP-type PGN-mediated activation of the imd pathway in vivo and in vitro (Kaneko et al., 2004; Leulier et al., 2003). The minimum structure of the DAP-type PGN required for PGRP-LC-mediated activation of the imd pathway is GlcNAcMurNAc with an internal 1,6-anhydro bond attached to a tripeptide containing DAP, known as tracheal cytotoxin (TCT) (Stenbak et al., 2004). PGRP-LE also recognizes TCT to activate the imd pathway and autophagy (Kaneko et al., 2006; Lim et al., 2006; Yano et al., 2008). Gramnegative bacteria release TCT or related short PGN fragments during cell wall remodeling following proliferation. Detection of PGN fragments allows flies to recognize the active growth of infecting bacteria. The PGRP-LC gene encodes three isomers, PGRP-LCa, -LCx, and LCy, which have identical intracellular domains and different extracellular PGRP domains with different specificities (Werner et al., 2000). The PGRP-LCx heterodimer, which has affinity for all PGN types, and PGRP-LCa, which has no affinity for PGN, act as a TCT receptor, whereas the PGRP-LCx homodimer complex acts as a DAP-type polymeric PGN receptor in vitro and in vivo (Kaneko et al., 2004; Mellroth et al., 2005; Stenbak et al., 2004). Crystal structure analysis revealed that binding of TCT to PGRP-LCx induces heterodimerization of PGRP-LCx and LCa, resulting in juxtaposition of the intracellular domains and signaling activation (Chang et al., 2006; Chang et al., 2005; Lim et al., 2006). Crystal structure analysis of TCT and PGRP-LE also demonstrated that the binding of TCT to PGRP-LE induces PGRP-LE multimerization (Lim et al., 2006). These results suggest that ligand binding to PGRPs induces receptor multimerization, 
such as PGRP-LCa/LCx, PGRP-LCx/LCx, PGRP-LE/LCx, and PGRP-LE/LE, which seems to be crucial for subsequent signaling activation. The DAP-type polymeric PGN and various PGN fragments are recognized in the extracellular space (hemolymph), on the cell surface, and in the intracelluler space (cytoplasm) by the dual detection system of PGRP-LE and -LC. Their presence may provide the host immune system an advantage for detecting various types (extracellular or intracellular) and states (static or proliferating) of bacteria having DAP-type PGN in the hemolymph, and various tissues and cells. A conserved motif, the RIP homotypic interaction motif (RHIM)-like motif, has been identified in the N-terminal signaling region of both PGRP-LC and -LE, and is crucial for activation of the imd pathway, although it is dispensable for interactions with the Imd adaptor protein (Kaneko et al., 2006). The conserved motif has similarity with the RHIM domain identified in adaptor proteins, RIP1 and TRIF, and is required for interaction between these two proteins and TRIF-dependent NF- $\kappa \mathrm{B}$ activation downstream of TLR3 in mammals (Meylan et al., 2004; Sun et al., 2002). In addition to imd pathway activation by ligand-dependent PGRP-LC and -LE multimerization, proteolysis of the extracellular domain of PGRP-LC is suggested to be involved in activation of the imd pathway (Schmidt et al., 2008; Warmbold et al., 2013).

The recognition of Lys-type PGN-containing bacteria by PGRP-SA, -SD, and GNBP1 upstream of the Toll pathway is relatively complicated. PGRP-SA preferentially binds to Lystype PGN and weakly binds to DAP-type PGN, consistent with the fact that the Toll pathway is activated in the fat body by Lys-type PGN and more weakly activated by DAP-type PGN (Chang et al., 2004; Mellroth et al., 2005). In fact, the minimum structure of the Lys-type PGN required for PGRP-SA-mediated activation of the Toll pathway comprises two units consisting of GlcNAc-MurNAc attached to Lys-containing tetrapeptides that are covalently dimerized by an interpeptide (muropeptide dimer) (Filipe et al., 2005). Recognition of many Lys-type PGN containing Gram-positive bacteria except Micrococcus luteus requires cooperation between PGRP-SA and PGRP-SD (Bischoff et al., 2004). The carboxypeptidase activity hydrolyzing DAP-type PGN of PGRP-SA is also suggested to suppress activation of the Toll pathway by DAP-type PGN (Chang et al., 2004). Moreover, GNBP1 is required for the PGRP-SA dependent recognition of Gram-positive bacteria (Wang et al., 2006). In addition to Lys-type PGN-binding activity, GNBP1 has muramidase-like activity and hydrolyzes Lys-type PGN to shorter muropeptides that are ligands for PGRP-SA (Wang et al., 2006). Therefore, the combination of 
PGRP-SA, -SD, and GNBP1 expands the recognized repertoire of Lys-type PGN-containing bacteria.

\subsection{Immunomodulating function of Drosophila PGRPs}

Some other PGRPs act as immune modulators in Drosophila. Five PGRPs, PGRP-LB, $\mathrm{SB} 1,-\mathrm{SB} 2$, -SC1(a/b), -SC2, are known or predicted to have amidase activity. The catalytic PGRPs cleave the amide bond connecting MurNAc to the Ala of the stem peptide. The degraded PGN loses its immunostimulatory activity. PGRP-LB, -SC1a, -SC1b, and -SC2 cleave DAPtype PGN, which modulates activation of the imd pathway (Bischoff et al., 2006; Mellroth et al., 2003; Paredes et al., 2011; Royet et al., 2011; Zaidman-Remy et al., 2006, Zaidman-Remy et al., 2011). These catalytic PGRPs also prevent excessive transfer of immunostimulatory PGN released into the hemolymph by commensal bacteria in the gut (Gendrin et al., 2009; Paredes et al., 2011). In tsetse flies, negative regulation of the imd pathway by PGRP-LB is important for etablishing immune tolerance against the symbiont Wigglesworthia glossinidia (Wang and Aksoy, 2012; Wang et al., 2009). The symbiosis is influenced by the symbiont-dependent induction of PGRP-LB degrading symbiotic PGN to inactive fragments in infected cells and the transmission of PGRP-LB to the offspring by the mother's milk. PGRP-LF utilizes the negative feedback regulation of the imd pathway in a different way (Basbous et al., 2011; Maillet et al., 2008; Persson et al., 2007; Werner et al., 2003;). PGRP-LF is a transmembrane PGRP with two PGRP domains lacking PGN binding ability, and it lacks the intracellular signaling domain. PGRP-LF binds to PGRP-LCx in the absence and presence of TCT and prevents formation of the PGRP-LCa/-LCx active heterodimer, which suppresses imd pathway activation.

In addition to its scavenger function, PGRP-SC1a is involved in bacterial phagocytosis (Garver et al., 2006). Extracellular PGRP-SC1a might bind to bacteria and enhance phagocytosis as an opsonin through phagocytic receptors, such as Eater. PGRP-SB1 has bactericidal activity (Mellroth and Steiner, 2006). Three of four mammalian PGRPs - PGLYRP1, PGLYRP3, and PGLYRP4 - also have bactericidal activity, but the bactericidal mechanisms differ between insect PGRP and mammalian PGRPs. The bactericidal activity of PGRP-SB1 is dependent on DAP-type PGN-specific amidase activity (Mellroth and Steiner, 2006), whereas mammalian PGRPs lack amidase activity and kill bacteria by the overactivation of bacterial two-component systems (Kashyap et al., 2011). 


\section{Conclusion and future perspectives}

Analysis of the role of PGRP family members in Drosophila immunity revealed that detection of PGN by PGRPs is the basis of the recognition of infectious bacteria and activation and modulation of immune responses. The sensor PGRPs - PGRP-SA, PGRP -SD, PGRP-LC, and PGRP-LE - distinguish the structural diversity of PGN and activate the appropriate immune responses, the Toll pathway, the imd pathway, the proPO cascade, and the autophagy in the hemolymph, on the cell surface, and in the cytoplasm of the fat body, the gut, and hemocytes. The catalytic PGRPs - PGRP-LB, -SB1, -SC1a, -SC1b, and -SC2 - modulate various immune responses by degrading PGN.

In mammals, some pattern recognition molecules recognize self-derived materials for the maintenance of homeostasis. For example, TLR4/MD-2, an LPS sensor, is stimulated by endogenous fatty acids, and is involved in metabolic diseases (Konner and Bruning, 2011). Inflammasomes are also activated by various self-derived materials (Henao-Mejia et al., 2012). It is not yet known, however, whether self-derived materials released from damaged tissues are recognized by Drosophila immune systems. Constitutive activation of the proPO cascade, which leads to localized wound healing and melanization, is induced by a mutation of Serpin 27A, a negative regulator of the proPO cascade, in the absence of infection. Connstitutive activation of the proPO cascade is suppressed by PGRP-LE mutation, suggesting that PGRP-LE is spontaneously stimulated to activate the proPO cascade in the absence of infection (Takehana et al., 2004). Further studies are required to examine whether pattern recognition molecules may be stimulated by self-derived materials for the maintenance of homeostasis in Drosophila.

\section{Funding}

This work was supported by Grants-in-Aid for Scientific Research from the Ministry of Education, Culture, Sports, Science, and Technology of Japan; the Japan Society for the Promotion of Science; Japan Science and Technology Agency; the Program for the Promotion of Basic Research Activities for Innovative Biosciences (PROBRAIN); the Strategic International Cooperative program from the Japan Science and Technology Agency; the National Institutes of Health (AI07495); the Takeda Science Foundation; the Mitsubishi Foundation; Astellas Foundation for Research on Metabolic Disorders; the Uehara Memorial Foundation; the Naito 
Foundation; Mochida Memorial Fundation; Terumo Life Science Foundation; Kowa Life Science Fundation; and a Global COE Research Grant (Tohoku University Ecosystem Adaptability).

\section{References}

Akira, S., Uematsu, S., Taekuchi, O., 2006. Pattern recognition and innateimmunity. Cell 124, 783-801.

Basbous, N., Coste, F., Leone, P., Vincentelli, R., Royet, J., Kellenberger, C., Roussel, A., 2011. The Drosophila peptidoglycan-recognition protein LF interacts with peptidoglycanrecognition protein LC to downregulate the Imd pathway. EMBO Rep. 12, 327-333.

Bischoff, V., Vignal, C., Boneca, I.G., Michel, T., Hoffmann, J.A., Royet, J., 2004. Function of the drosophila pattern-recognition receptor PGRP-SD in the detection of Gram-positive bacteria. Nat. Immunol. 5, 1175-1180.

Bischoff, V., Vignal, C., Duvic, B., Boneca, I.G., Hoffmann, J.A., Royet, J., 2006. Downregulation of the Drosophila immune response by peptidoglycan-recognition proteins SC1 and SC2. PLoS Pathog. 2, e14.

Bosco-Drayon, V., Poidevin, M., Boneca, I.G., Narbonne-Reveau, K., Royet, J., Charroux, B., 2012. Peptidoglycan sensing by the receptor PGRP-LE in the Drosophila gut induces immune responses to infectious bacteria and tolerance to microbiota. Cell Host Microbe 12, 153-165.

Chang, C.I., Chelliah, Y., Borek, D., Mengin-Lecreulx, D., Deisenhofer, J., 2006. Structure of tracheal cytotoxin in complex with a heterodimeric pattern-recognition receptor. Science 311, 1761-1764.

Chang, C.I., Ihara, K., Chelliah, Y., Mengin-Lecreulx, D., Wakatsuki, S., Deisenhofer, J., 2005. 
Structure of the ectodomain of Drosophila peptidoglycan-recognition protein LCa suggests a molecular mechanism for pattern recognition. Proc. Natl. Acad. Sci. U. S. A. 102, 10279-10284.

Chang, C.I., Pili-Floury, S., Herve, M., Parquet, C., Chelliah, Y., Lemaitre, B., Mengin-Lecreulx, D., Deisenhofer, J., 2004. A Drosophila pattern recognition receptor contains a peptidoglycan docking groove and unusual L,D-carboxypeptidase activity. PLoS Biol. 2, E277.

Choe, K.M., Werner, T., Stoven, S., Hultmark, D., Anderson, K.V., 2002. Requirement for a peptidoglycan recognition protein (PGRP) in Relish activation and antibacterial immune responses in Drosophila. Science 296, 359-362.

Christophides G.K. et al., 2002. Immunity-related genes and gene families in Anopheles gambiae. Science 298, 159-165.

Ferrandon, D., Imler, J.L., Hetru, C., Hoffmann, J.A., 2007. The Drosophila systemic immune response: sensing and signalling during bacterial and fungal infections. Nat. Rev. Immunol. 7, 862-874.

Filipe, S.R., Tomasz, A., Ligoxygakis, P., 2005. Requirements of peptidoglycan structure that allow detection by the Drosophila Toll pathway. EMBO Rep. 6, 327-333.

Garver, L.S., Wu, J., Wu, L.P., 2006. The peptidoglycan recognition protein PGRP-SC1a is essential for Toll signaling and phagocytosis of Staphylococcus aureus in Drosophila. Proc. Natl. Acad. Sci. U. S. A. 103, 660-665.

Gendrin, M., Welchman, D.P., Poidevin, M., Herve, M., Lemaitre, B., 2009. Long-range activation of systemic immunity through peptidoglycan diffusion in Drosophila. PLoS Pathog. 5, e1000694. 
Gobert, V., Gottar, M., Matskevich, A.A., Rutschmann, S., Royet, J., Belvin, M., Hoffmann, J.A., Ferrandon, D., 2003. Dual activation of the Drosophila toll pathway by two pattern recognition receptors. Science 302, 2126-2130.

Goto, A., Yano, T., Terashima, J., Iwashita, S., Oshima, Y., Kurata, S., 2010. Cooperative regulation of the induction of the novel antibacterial Listericin by peptidoglycan recognition protein LE and the JAK-STAT pathway. J. Biol. Chem. 285, 15731-15738.

Gottar, M., Gobert, V., Matskevich, A.A., Reichhart, J.M., Wang, C., Butt, T.M., Belvin, M., Hoffmann, J.A., Ferrandon, D., 2006. Dual detection of fungal infections in Drosophila via recognition of glucans and sensing of virulence factors. Cell 127, 1425-1437.

Gottar, M., Gobert, V., Michel, T., Belvin, M., Duyk, G., Hoffmann, J.A., Ferrandon, D., Royet, J., 2002. The Drosophila immune response against Gram-negative bacteria is mediated by a peptidoglycan recognition protein. Nature 416, 640-644.

Guan, R., Mariuzza, R.A., 2007. Peptidoglycan recognition proteins of the innate immune system. Trends Microbiol. 15, 127-134.

Henao-Mejia, J., Elinav, E., Strowig, T., Flavell, R.A., 2012. Inflammasomes: far beyond inflammation. Nat. Immunol. 13, 321-324.

Hoffmann, J.A., Reichhart, J.M., 2002. Drosophila innate immunity: an evolutionary perspective. Nat. Immunol. 3, 121-126.

Hultmark, D., 2003. Drosophila immunity: paths and patterns. Curr. Opin. Immunol. 15, 12-19.

Jang, I.H., Chosa, N., Kim, S.H., Nam, H.J., Lemaitre, B., Ochiai, M., Kambris, Z., Brun, S., Hashimoto, C., Ashida, M., Brey, P.T., Lee, W.J., 2006. A Spatzle-processing enzyme required for toll signaling activation in Drosophila innate immunity. Dev. Cell 10, 45-55.

Kaneko, T., Goldman, W.E., Mellroth, P., Steiner, H., Fukase, K., Kusumoto, S., Harley, W., Fox, 
A., Golenbock, D., Silverman, N., 2004. Monomeric and polymeric gram-negative peptidoglycan but not purified LPS stimulate the Drosophila IMD pathway. Immunity 20, 637-649.

Kaneko, T., Yano, T., Aggarwal, K., Lim, J.H., Ueda, K., Oshima, Y., Peach, C., Erturk-Hasdemir, D., Goldman, W.E., Oh, B.H., Kurata, S., Silverman, N., 2006. PGRP-LC and PGRP-LE have essential yet distinct functions in the drosophila immune response to monomeric DAP-type peptidoglycan. Nat. Immunol. 7, 715-723.

Kang, D., Liu, G., Lundstrom, A., Gelius, E., Steiner, H., 1998. A peptidoglycan recognition protein in innate immunity conserved from insects to humans. Proc. Natl. Acad. Sci. U. S. A. $95,10078-10082$.

Kashyap, D.R., Wang, M., Liu, L.H., Boons, G.J., Gupta, D., Dziarski, R., 2011. Peptidoglycan recognition proteins kill bacteria by activating protein-sensing two-component systems. Nat. Med. 17, 676-683.

Kim, C.H., Park, J.W., Ha, N.C., Kang, H.J., Lee, B.L., 2008. Innate immune response in insects: recognition of bacterial peptidoglycan and amplification of its recognition signal. BMB Rep. 41, 93-101.

Kim, M.S., Byun, M., Oh, B.H., 2003. Crystal structure of peptidoglycan recognition protein LB from Drosophila melanogaster. Nat. Immunol. 4, 787-793.

Konner, A.C., Bruning, J.C., 2011. Toll-like receptors: linking inflammation to metabolism. Trends Endocrinol. Metab. 22, 16-23.

Lee, W.J., Lee, J.D., Kravchenko, V.V., Ulevitch, R.J., Brey, P.T., 1996. Purification and molecular cloning of an inducible gram-negative bacteria-binding protein from the silkworm, Bombyx mori. Proc. Natl. Acad. Sci. U. S. A. 93, 7888-7893. 
Lemaitre, B., Hoffmann, J., 2007. The host defense of Drosophila melanogaster. Annu. Rev. Immunol. 25, 697-743.

Lemaitre, B., Reichhart, J.M., Hoffmann, J.A., 1997. Drosophila host defense: differential induction of antimicrobial peptide genes after infection by various classes of microorganisms. Proc. Natl. Acad. Sci. U. S. A. 94, 14614-14619.

Leulier, F., Parquet, C., Pili-Floury, S., Ryu, J.H., Caroff, M., Lee, W.J., Mengin-Lecreulx, D., Lemaitre, B., 2003. The Drosophila immune system detects bacteria through specific peptidoglycan recognition. Nat. Immunol. 4, 478-484.

Ligoxygakis, P., Pelte, N., Hoffmann, J.A., Reichhart, J.M., 2002. Activation of Drosophila Toll during fungal infection by a blood serine protease. Science 297, 114-116.

Lim, J.H., Kim, M.S., Kim, H.E., Yano, T., Oshima, Y., Aggarwal, K., Goldman, W.E., Silverman, N., Kurata, S., Oh, B.H., 2006. Structural basis for preferential recognition of diaminopimelic acid-type peptidoglycan by a subset of peptidoglycan recognition proteins. J. Biol. Chem. 281, 8286-8295.

Maillet, F., Bischoff, V., Vignal, C., Hoffmann, J., Royet, J., 2008. The Drosophila peptidoglycan recognition protein PGRP-LF blocks PGRP-LC and IMD/JNK pathway activation. Cell Host Microbe 3, 293-303.

Medzhitov, R., Janeway, C.A., Jr., 2002. Decoding the patterns of self and nonself by the innate immune system. Science 296, 298-300.

Mellroth, P., Karlsson, J., Hakansson, J., Schultz, N., Goldman, W.E., Steiner, H., 2005. Ligandinduced dimerization of Drosophila peptidoglycan recognition proteins in vitro. Proc. Natl. Acad. Sci. U. S. A. 102, 6455-6460.

Mellroth, P., Karlsson, J., Steiner, H., 2003. A scavenger function for a Drosophila peptidoglycan 
recognition protein. J. Biol. Chem. 278, 7059-7064.

Mellroth, P., Steiner, H., 2006. PGRP-SB1: an N-acetylmuramoyl L-alanine amidase with antibacterial activity. Biochem. Biophys. Res. Commun. 350, 994-999.

Meylan, E., Burns, K., Hofmann, K., Blancheteau, V., Martinon, F., Kelliher, M., Tschopp, J., 2004. RIP1 is an essential mediator of Toll-like receptor 3-induced NF-kappa B activation. Nat. Immunol. 5, 503-507.

Michel, T., Reichhart, J.M., Hoffmann, J.A., Royet, J., 2001. Drosophila Toll is activated by Gram-positive bacteria through a circulating peptidoglycan recognition protein. Nature 414, 756-759.

Neyen, C., Poidevin, M., Roussel, A., Lemaitre, B., 2012. Tissue- and ligand-specific sensing of gram-negative infection in drosophila by PGRP-LC isoforms and PGRP-LE. J. Immunol. 189, 1886-1897.

Paredes, J.C., Welchman, D.P., Poidevin, M., Lemaitre, B., 2011. Negative regulation by amidase PGRPs shapes the Drosophila antibacterial response and protects the fly from innocuous infection. Immunity 35, 770-779.

Persson, C., Oldenvi, S., Steiner, H., 2007. Peptidoglycan recognition protein LF: a negative regulator of Drosophila immunity. Insect Biochem. Mol. Biol. 37, 1309-1316.

Ramet, M., Manfruelli, P., Pearson, A., Mathey-Prevot, B., Ezekowitz, R.A., 2002. Functional genomic analysis of phagocytosis and identification of a Drosophila receptor for E. coli. Nature 416, 644-648.

Royet, J., Gupta, D., Dziarski, R., 2011. Peptidoglycan recognition proteins: modulators of the microbiome and inflammation. Nat. Rev. Immunol. 11, 837-851.

Schleifer, K.H., Kandler, O., 1972. Peptidoglycan types of bacterial cell walls and their 
taxonomic implications. Bacteriol. Rev. 36, 407-477.

Schmidt, R.L., Trejo, T.R., Plummer, T.B., Platt, J.L., Tang, A.H., 2008. Infection-induced proteolysis of PGRP-LC controls the IMD activation and melanization cascades in Drosophila. FASEB J. 22, 918-929.

Soderhall, K., Cerenius, L., 1998. Role of the prophenoloxidase-activating system in invertebrate immunity. Curr. Opin. Immunol. 10, 23-28.

Steiner, H., Hultmark, D., Engstrom, A., Bennich, H., Boman, H.G., 2009. Sequence and specificity of two antibacterial proteins involved in insect immunity. Nature 292: 246-248. 1981. J. Immunol. 182, 6635-6637.

Stenbak, C.R., Ryu, J.H., Leulier, F., Pili-Floury, S., Parquet, C., Herve, M., Chaput, C., Boneca, I.G., Lee, W.J., Lemaitre, B., Mengin-Lecreulx, D., 2004. Peptidoglycan molecular requirements allowing detection by the Drosophila immune deficiency pathway. J. Immunol. 173, 7339-7348.

Sun, X., Yin, J., Starovasnik, M.A., Fairbrother, W.J., Dixit, V.M., 2002. Identification of a novel homotypic interaction motif required for the phosphorylation of receptor-interacting protein (RIP) by RIP3. J. Biol. Chem. 277, 9505-9511.

Takehana, A., Katsuyama, T., Yano, T., Oshima, Y., Takada, H., Aigaki, T., Kurata, S., 2002. Overexpression of a pattern-recognition receptor, peptidoglycan-recognition protein-LE, activates imd/relish-mediated antibacterial defense and the prophenoloxidase cascade in Drosophila larvae. Proc. Natl. Acad. Sci. U. S. A. 99, 13705-13710.

Takehana, A., Yano, T., Mita, S., Kotani, A., Oshima, Y., Kurata, S., 2004. Peptidoglycan recognition protein (PGRP)-LE and PGRP-LC act synergistically in Drosophila immunity. EMBO J. 23, 4690-4700. 
Travassos, L.H., Carneiro, L.A., Ramjeet, M., Hussey, S., Kim, Y.G., Magalhaes, J.G., Yuan, L., Soares, F., Chea, E., Le Bourhis, L., Boneca, I.G., Allaoui, A., Jones, N.L., Nunez, G., Girardin, S.E., Philpott, D.J., 2010. Nod1 and Nod2 direct autophagy by recruiting ATG16L1 to the plasma membrane at the site of bacterial entry. Nat. Immunol. 11, 55-62.

Wang, J., Aksoy, S., 2012. PGRP-LB is a maternally transmitted immune milk protein that influences symbiosis and parasitism in tsetse's offspring. Proc. Natl. Acad. Sci. U. S. A. 109, 10552-10557.

Wang, J., Wu, Y., Yang, G., Aksoy, S., 2009. Interactions between mutualist Wigglesworthia and tsetse peptidoglycan recognition protein (PGRP-LB) influence trypanosome transmission. Proc. Natl. Acad. Sci. U. S. A. 106, 12133-12138.

Wang, L., Weber, A.N., Atilano, M.L., Filipe, S.R., Gay, N.J., Ligoxygakis, P., 2006. Sensing of Gram-positive bacteria in Drosophila: GNBP1 is needed to process and present peptidoglycan to PGRP-SA. EMBO J. 25, 5005-5014.

Warmbold, C., Uliczka, K., Rus, F., Suck, R., Petersen, A., Silverman, N., Ulmer, A.J., Heine, H., Roeder, T., 2013. Dermatophagoides pteronyssinus major allergen 1 activates the innate immune response of the fruit fly Drosophila melanogaster. J. Immunol. 190, 366-371.

Weber, A.N., Tauszig-Delamasure, S., Hoffmann, J.A., Lelievre, E., Gascan, H., Ray, K.P., Morse, M.A., Imler, J.L., Gay, N.J., 2003. Binding of the Drosophila cytokine Spatzle to Toll is direct and establishes signaling. Nat. Immunol. 4, 794-800.

Werner, T., Liu, G., Kang, D., Ekengren, S., Steiner, H., Hultmark, D., 2000. A family of peptidoglycan recognition proteins in the fruit fly Drosophila melanogaster. Proc. Natl. Acad. Sci. U. S. A. 97, 13772-13777.

Werner T., Borge-Renberg, K., Mellroth, P., Steiner, H., Hultmark, D., 2003. Functional 
diversity of the Drosophila PGRP-LC gene cluster in the response to lipopolysaccharide and peptidoglycan. J. Biol. Chem. 278, 26319-26322.

Yano, T., Mita, S., Ohmori, H., Oshima, Y., Fujimoto, Y., Ueda, R., Takada, H., Goldman, W.E., Fukase, K., Silverman, N., Yoshimori, T., Kurata, S., 2008. Autophagic control of listeria through intracellular innate immune recognition in drosophila. Nat. Immunol. 9, 908-916.

Yoshida, H., Kinoshita, K., Ashida, M., 1996. Purification of a peptidoglycan recognition protein from hemolymph of the silkworm, Bombyx mori. J. Biol. Chem. 271, 13854-13860.

Zaidman-Remy, A., Herve, M., Poidevin, M., Pili-Floury, S., Kim, M.S., Blanot, D., Oh, B.H., Ueda, R., Mengin-Lecreulx, D., Lemaitre, B., 2006. The Drosophila amidase PGRP-LB modulates the immune response to bacterial infection. Immunity 24, 463-473.

Zaidman-Rémy, A., Poidevin, M., Hervé, M., Welchman, D.P., Paredes, J.C., Fahlander, C., Steiner, H., Mengin-Lecreulx, D., Lemaitre, B. 2011. Drosophila immunity: analysis of PGRP-SB1 expression, enzymatic activity and function. PLoS One. 6, e17231.

\section{Figure legends}

\section{Figure 1. Peptidoglycan structure.}

Peptidoglycans (PGN) are polymers of $\beta$-1,4-linked $N$-acetylglucosamine (GlcNAc) and $N$ acetylmuramic acid (MurNAc) cross-linked by short stem peptides, and are categorized into two major types: Lys-type (a) and diaminopimelic acid (DAP)-type (b), based on the amino acid composition of the stem peptides and the linkage between the stem peptides.

\section{Figure 2. Structure of peptidoglycan recognition proteins in Drosophila}

Conserved domains and main functions are represented. Courtesy of T. Yano.

\section{Figure 3. Function of peptidoglycan recognition proteins in Drosophila}


Peptidoglycan recognition protein (PGRP)-SA in the hemolymph binds to Lys-type PGN and is required for activation of the Toll pathway in cooperation with PGRP-SD and Gram-negative binding protein (GNBP)1. GNBP3 is involved in yeast-mediated activation of the Toll pathway. Activation of the Toll pathway is mediated by its active ligand, Spätzle (Spz), cleaved from proSpätzle by the serine protease cascade, including the Spz-processing enzyme (SPE). Upstream of the imd pathway, the PGRP-LCx homodimer complex acts as a DAP-type polymeric PGN receptor, and the heterodimer of PGRP-LCx and -LCa acts as a receptor for DAP-type monomeric PGN, such as tracheal cytotoxin (TCT). PGRP-LE binds to polymeric and monomeric DAP-type PGN. Extracellular PGRP-LE activates the imd pathway through PGRPLC transmembrane receptors and is involved in activation of the prophenoloxidase (proPO) cascade upstream of the proPO-activating enzyme (PPAE), which is regulated by a serine protease inhibitor, Serpin 27A. PGRP-LF with two PGRP domains lacking PGN-binding ability binds to PGRP-LCx and prevents the formation of the PGRP-LC active dimer, which suppresses the activation of the imd pathway. Intracellular PGRP-LE recognizes intracellular bacteria with DAP-type PGN and activates the imd pathway through Imd adaptor protein and autophagy in an imd pathway-independent manner. PGRP-LB and -SC1a/1b/2 cleave DAP-type PGN to inactive fragments, which suppresses activation of the imd pathway. In addition to its scavenger function, PGRP-SC1a is involved in the phagocytosis of bacteria as an opsonin. PGRP-SB1 has DAP-type PGN-specific amidase-dependent bactericidal activity. 
$\begin{array}{ll}\text { a. Lys-type PGN b. DAP-type PGN } & \text { b. }\end{array}$ $\beta 1 \rightarrow 4$ GICNAC $\beta 1 \rightarrow 4$ GICNAC $\beta 1 \rightarrow 4$ MurNAc $\quad \beta 1 \rightarrow 4 /$ GlcNAc $_{\beta 1} \rightarrow 4 /$ MurNAc $\quad \beta 1 \rightarrow 4 /$ GlcNAc GICNAC L-Ala $\beta 1 \rightarrow 4$ MurNAc, GICNAC L-Ala $\beta 1 \rightarrow 4$ MurNAc

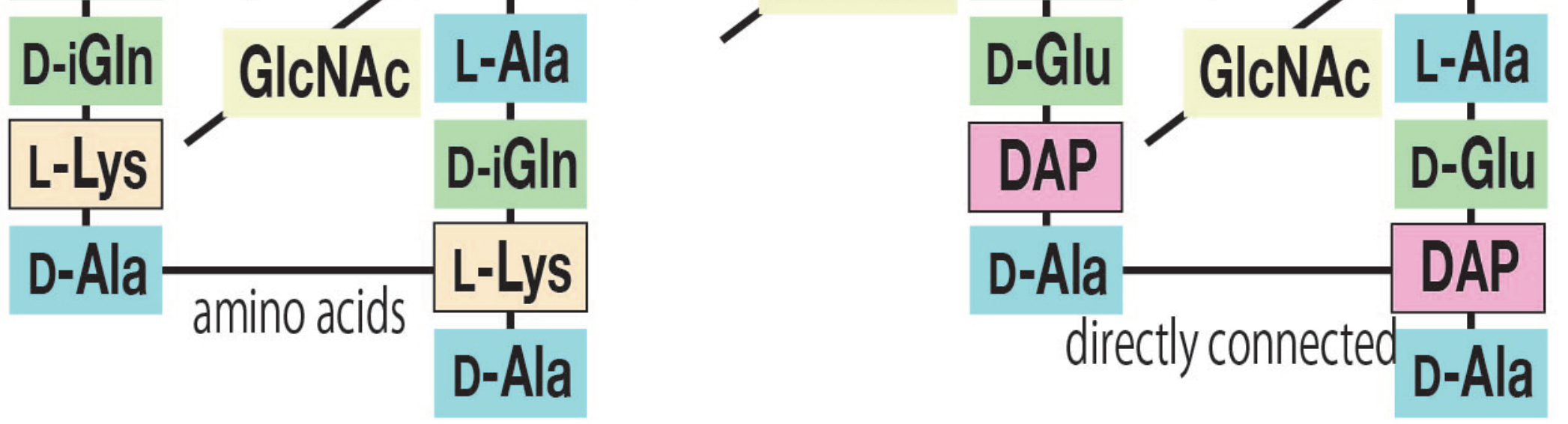




\section{Drosophila PGRPs}

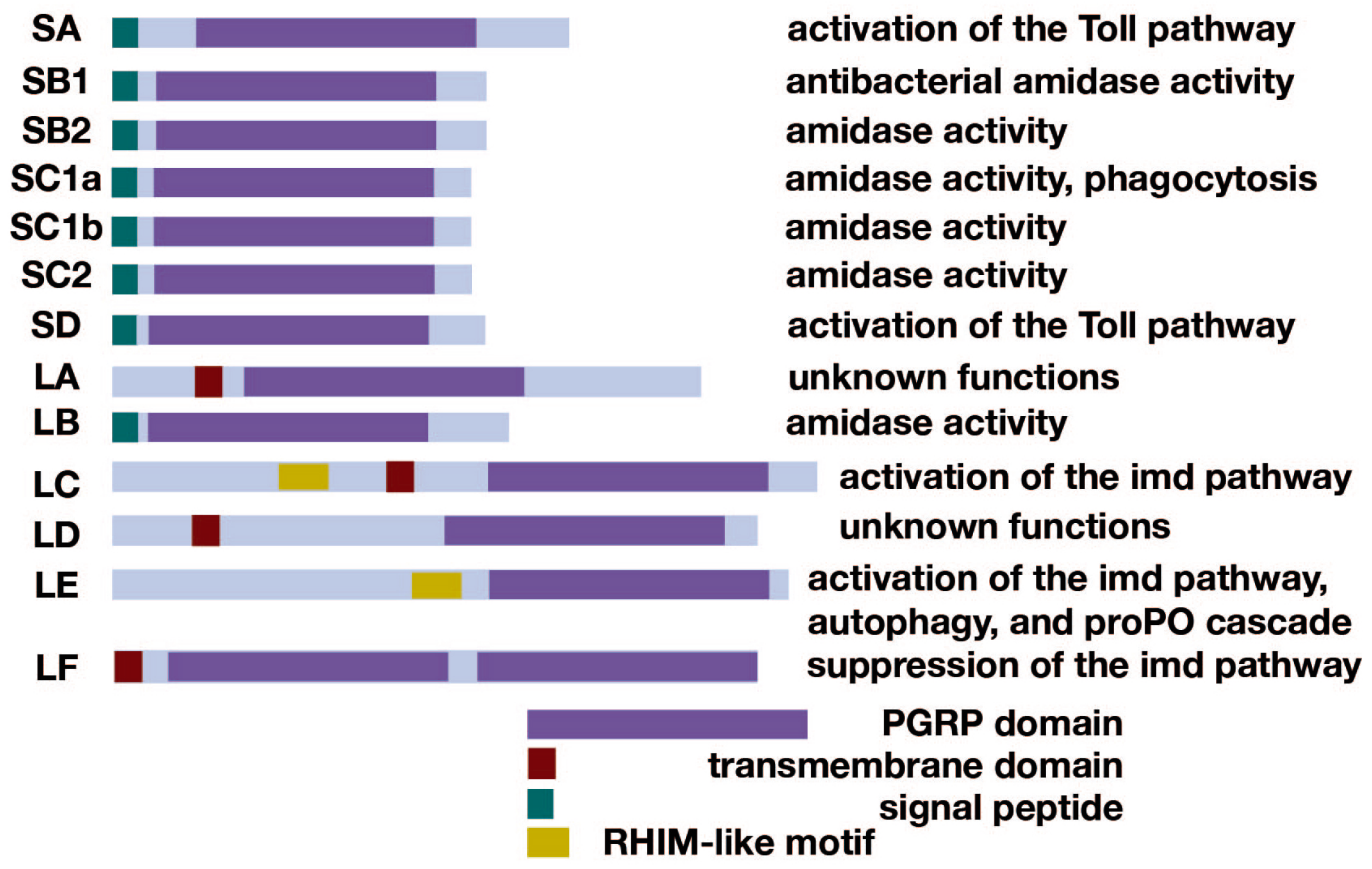




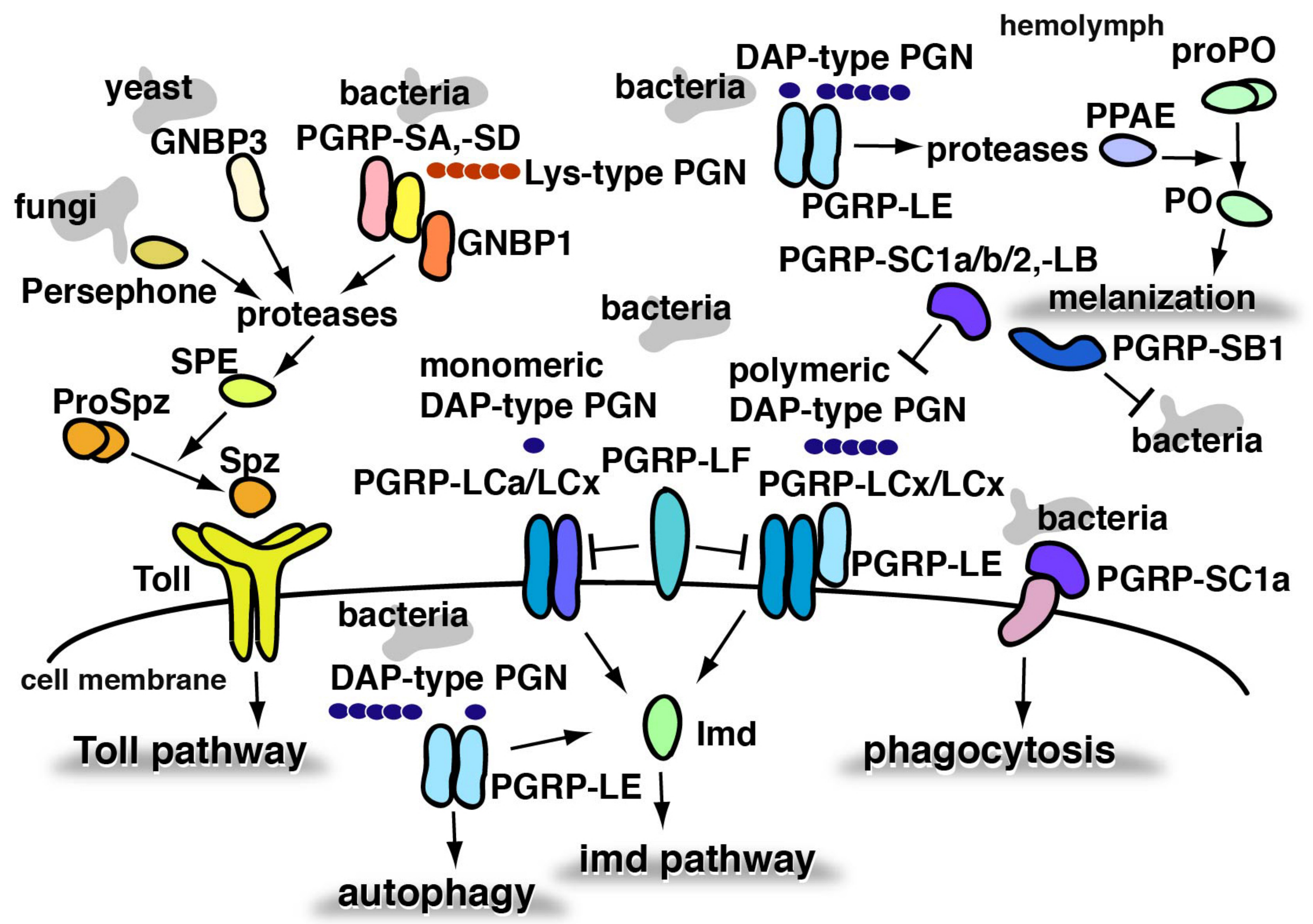

